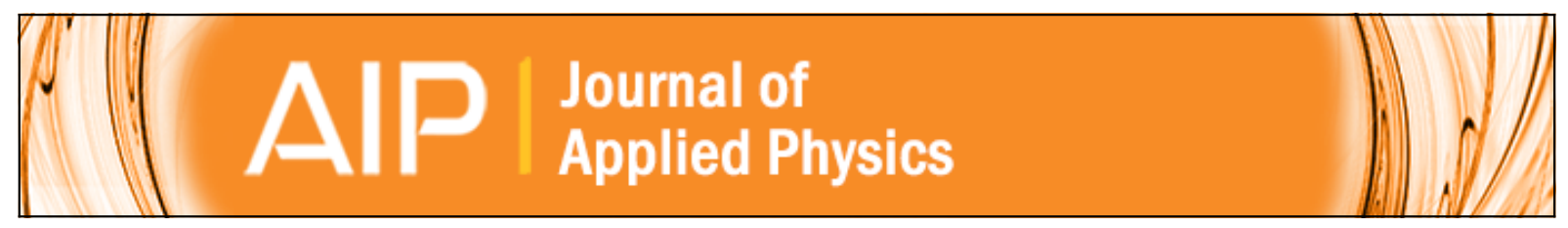

\title{
Surface morphology and optical properties of thin films of thiophene-based binary blends
}

Marco Salerno, Robert lan Renton Blyth, Julie Thompson, Roberto Cingolani, and Giuseppe Gigli

Citation: Journal of Applied Physics 98, 013512 (2005); doi: 10.1063/1.1944215

View online: http://dx.doi.org/10.1063/1.1944215

View Table of Contents: http://scitation.aip.org/content/aip/journal/jap/98/1?ver=pdfcov

Published by the AIP Publishing

\section{Articles you may be interested in}

Structural, morphological, FTIR and photoluminescence properties of gallium oxide thin films

J. Vac. Sci. Technol. B 32, 03D119 (2014); 10.1116/1.4868523

$\mathrm{ZnO}$ thin film deposition using colliding plasma plumes and single plasma plume: Structural and optical properties

J. Appl. Phys. 114, 224903 (2013); 10.1063/1.4846115

Optical and transient photoconductive properties of pentacene and functionalized pentacene thin films:

Dependence on film morphology

J. Appl. Phys. 98, 033701 (2005); 10.1063/1.1949711

Structural and optical properties of 6,13-pentacenequinone thin films

Appl. Phys. Lett. 85, 5568 (2004); 10.1063/1.1832759

Morphology and electronic transport of polycrystalline pentacene thin-film transistors

Appl. Phys. Lett. 82, 3907 (2003); 10.1063/1.1578536

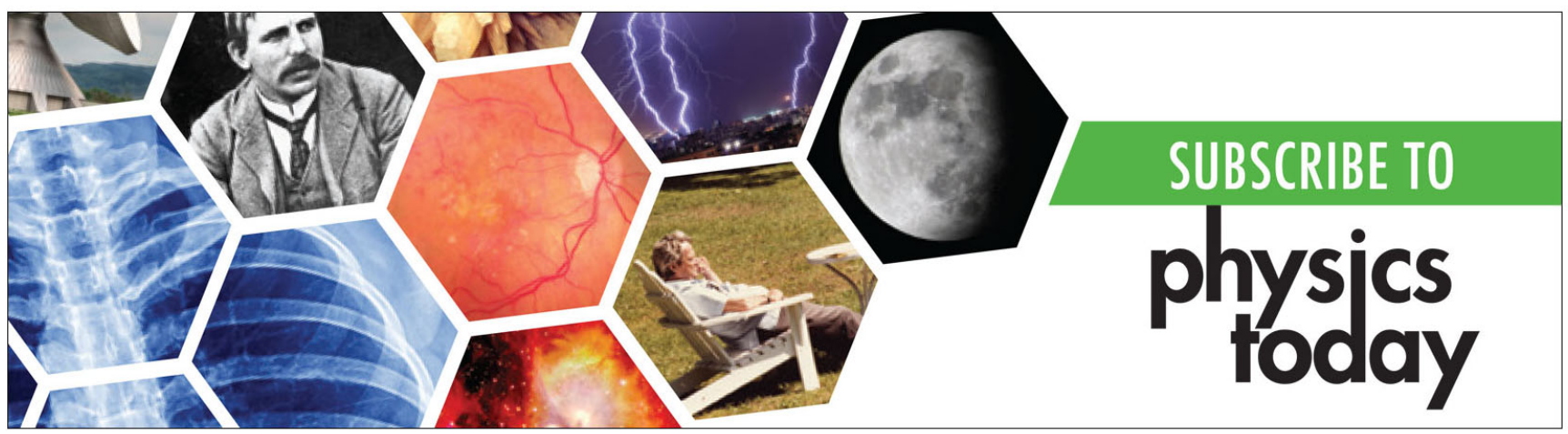




\title{
Surface morphology and optical properties of thin films of thiophene-based binary blends
}

\author{
Marco Salerno, ${ }^{\text {a) }}$ Robert lan Renton Blyth, Julie Thompson, Roberto Cingolani, and \\ Giuseppe Gigli \\ National Nanotechnology Laboratory of Istituto Nazionale per la Fisica della Materia, \\ via Arnesano km 5, I-73100 Lecce, Italy
}

(Received 17 January 2005; accepted 8 May 2005; published online 6 July 2005)

\begin{abstract}
We report on the relationship between optical and morphological properties of thin films of a blend of thiophene-based monomer and a diamine derivative. This system is of interest as it gives rise to white emission, due to the formation of exciplex energy levels in the solid state. The photoluminescence emission has been collected for different relative concentrations of the two blend components, and the film topography has been mapped correspondingly by means of atomic force microscopy. Along with a complex evolution of the film surface morphology, a significant variation of the emission properties has been observed upon change of the blend composition. In particular, the intensity of the broad low-energy exciplex band turns out to be affected by a balance of two factors, namely, the extension of homogeneous blend film areas, and their effective concentration. (C) 2005 American Institute of Physics. [DOI: 10.1063/1.1944215]
\end{abstract}

\section{INTRODUCTION}

The photo- and electroluminescence properties of thin films of organic compounds have been extensively investigated in the last decade, due to the increasing interest in organic light-emitting devices (OLEDs). ${ }^{1-3}$ The main advantage of the "organic route" to semiconductor materials is the possibility to fabricate large area, low cost devices. More recently, attention has moved from backlighting and display applications to general illumination, for which generation of white light is required such as in white organic light-emitting devices (WOLEDs). ${ }^{4-6}$ One method to obtain white light from OLEDs is the so-called bulk exciplex approach, which relies on the recombination from intermolecular electronic levels formed in a molecular blend. ${ }^{7,8}$ Compared to multilayer structures and blends of materials emitting different primary colors 9,10 the "exciplex" method has the advantage of a negligible dependence of the electroluminescence on the applied voltage. ${ }^{11}$ This turns out to be fundamental in applications where power intensity, but not color, has to be modulated. On the other hand, a deep understanding of the correlation between the structural and optical properties is crucial in these systems for controlling the device properties. The morphology of molecular materials following deposition is known to be characterized by labile equilibrium states, ${ }^{12}$ whose change can strongly affect the emissive properties. This is especially the case for thin films, where critical boundary conditions apply, and for mixtures of heavy molecules of low compatibility. ${ }^{13-15}$

In this work we present a detailed atomic force microscopy (AFM) study of spin-coated films of a blend of materials used as the active layers in the fabrication of WOLEDs, ${ }^{16}$ namely, 2,5-bis(trimethylsilyl)-thiophene-1,1-

\footnotetext{
a) Author to whom correspondence should be addressed; electronic mail: marco.salerno@unile.it
}

dioxide $\quad(\mathrm{STO})^{17,18}$ and $N, N^{\prime}$-bis(3-methylphenyl)$N, N^{\prime}$-diphenylbenzidine (TPD). ${ }^{19}$ The respective molecular structures are reported in Fig. 1.

\section{EXPERIMENT}

STO [molecular weight $\left.\left(\mathrm{M}_{\mathrm{w}}\right)=260.54 \mathrm{amu}\right]$ was synthesized and purified according to Ref. 17, while TPD $\left(\mathrm{M}_{\mathrm{w}}\right.$ $=516.67 \mathrm{amu}$ ) was acquired from Aldrich. The two molecular compounds were blended together into toluene solutions of a 50-mM total concentration, while varying the relative molar concentration of STO $(\Phi)$ from $0 \%$ to $100 \%$ in $10 \%$ steps. The blend solutions were spin cast onto UVtransparent glass substrates, at a speed of $2000 \mathrm{rpm}$ for $1 \mathrm{~min}$. No thermal curing was adopted, but solvent evaporation was allowed to occur at room temperature.

Within $10 \mathrm{~min}$ after preparation, all the films have been examined in a Cary Eclipse fluorescence spectrometer for photoluminescence (PL) measurements. Soon after the optical characterization, the same samples were moved to the AFM for observation of their surface morphology. These measurements have been carried out by means of a commer-

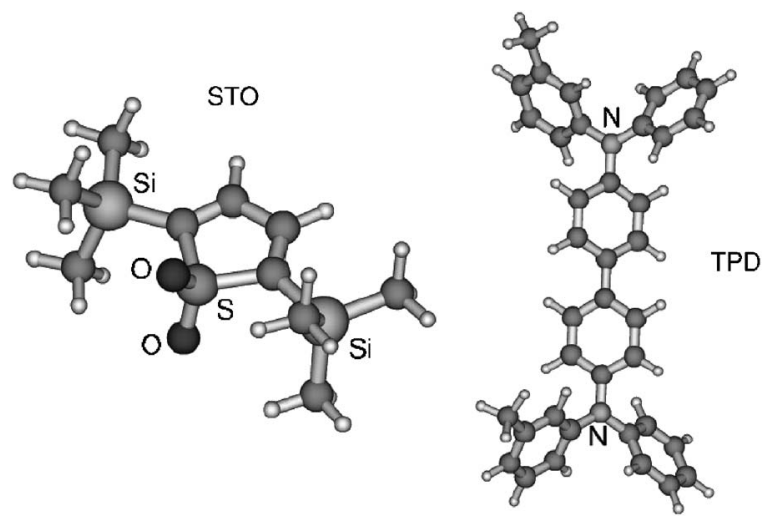

FIG. 1. Molecular structures of STO and TPD. 


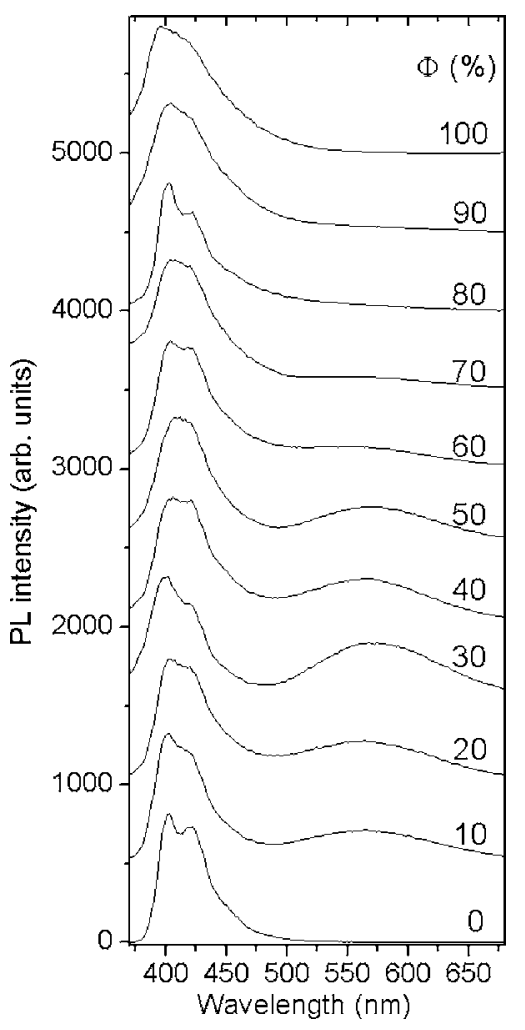

FIG. 2. PL emission spectra of all the STO/TPD blend films, normalized to the maximum intensity of the blue peak, for different $\Phi$ values (see inset).

cial AFM by NT-MDT, operating in tapping mode. Silicon cantilevers were used with nominal spring constant and resonance frequency of $15 \mathrm{~N} / \mathrm{m}$ and $260 \mathrm{kHz}$, respectively, at a free oscillation amplitude of about $200 \mathrm{~nm}$ and a relative set point amplitude of $60 \%$.

\section{OPTICAL RESULTS}

In Fig. 2 the PL spectra for an excitation wavelength of $350 \mathrm{~nm}$ are reported. In most cases two features are observed. First, at shorter wavelengths a structured band associated with the emission from the individual blend components shows up. In fact, the PL emissions expected from the two populations of TPD and STO molecules largely overlap, with maxima around 400 and $420 \mathrm{~nm}$ wavelengths, respectively. ${ }^{19}$ Second, at longer wavelengths, in the red region of the visible spectrum, a broadband peaked at about $540 \mathrm{~nm}$ appears, due to the emission from intermolecular TPD and STO exciplex states. ${ }^{11,19}$ The combination of the "blue" peak from isolated molecules and the "red" exciplex band has been demonstrated to give rise to bright white emission in the solid state. ${ }^{11,20}$

The intensity of the exciplex band changes significantly with $\Phi$, whereas the position of its maximum shows a comparatively little variation (spanned range about $7 \mathrm{~nm}$ ). In Fig. 3(a) the maximum intensity of the PL exciplex band, normalized to the maximum of the blue emission peak, has been plotted versus $\Phi$. For this plot, in the cases where no exciplex band is evident $(\Phi=0 \%, 80 \%, 90 \%$, and $100 \%$, see Fig. 2 ) the intensity at the average position of the exciplex maximum in the other spectra (wavelength equal to $569 \mathrm{~nm}$ ) is
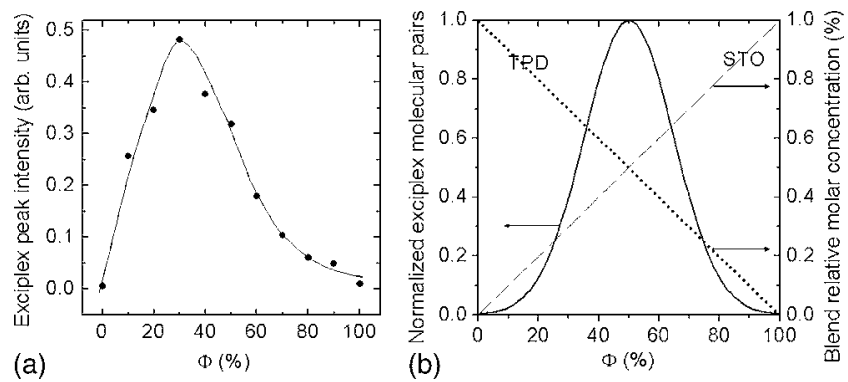

FIG. 3. (a) Experimental values of the maximum intensity of the exciplex PL band versus $\Phi$. The line is just a guide to the eye. (b) Ideal model of the blend film system. Solid line: expected normalized profile of the number of heterogeneous molecular pairs in the blend vs $\Phi$; dashed and dotted lines: relative molar concentration of the two blend components STO and TPD vs $\Phi$, respectively.

reported. No exciplex emission is obviously observed when only one component of the blend is present $(\Phi=0 \%$ and $100 \%$ ), whereas a maximum intensity occurs for $\Phi=30 \%$. In fact, in the case of a homogeneous distribution of the two molecular species in the blend film, $\Phi=50 \%$ should be the situation for which the number of closely spaced heterogeneous pairs of molecules (one of STO and one of TPD), likely giving rise to exciplex states, is maximized. This ideal situation is schematically represented in Fig. 3(b). Therefore, in the presence of such a disagreement of the maximum exciplex position versus $\Phi$, structural effects, with the blend surface deviating from the continuous and uniform film morphology, are to be expected.

\section{MORPHOLOGICAL RESULTS}

In Fig. 4 the representative AFM images of the surface morphology of STO/TPD blend films at different relative molar STO concentrations $\Phi$ are reported. At $\Phi=0 \%$ [Fig. 4(a)] the pure TPD sample looks flat and smooth. Only on a very compressed height scale (1 $\mathrm{nm}$ full range) some roughness, and local defects of the film are evident. The same also holds for the $\Phi=10 \%$ film (not reported). At $\Phi=20 \%$ [Fig. 4(b)] with the increasing amount of STO, holes appear in the film (mean diameter of $320 \mathrm{~nm}$ and mean density of $1.4 \mu \mathrm{m}^{-2}$ ). These are probably due to the interaction between molecules of different species, which make the film dewet the substrate in uniformly distributed points. At $\Phi=30 \%$ [Fig. 4(c)] extended islands appear on a generally flat background. This lower background level is still a molecular film and not the bare glass substrate, as was checked in different regions by making scratches with a needle. Due to the peculiar shape of the borders and to the rather uniform height $(20 \pm 5 \mathrm{~nm})$, the islands can be attributed to crystallization of one of the molecular species, generating partial phase separation of the blend. At $\Phi=40 \%$ a few crystalline islands are still present, but their degree of coverage is much lower (around 5\% against $40 \%$ of the $\Phi=30 \%$ case) and most of the sample surface is as uniform as that reported in Fig. 4(d). There, holes appear again as for $\Phi=20 \%$, but slightly larger (mean diameter of $400 \mathrm{~nm}$ ) and with lower density (around $0.8 \mu \mathrm{m}^{-2}$ ). At $\Phi=50 \%$ [Fig. 4(e)] no more crystalline islands appear, and the number of holes and their size return to values similar to the $20 \%$ sample [Fig. 4(b)]. Yet, depressed 


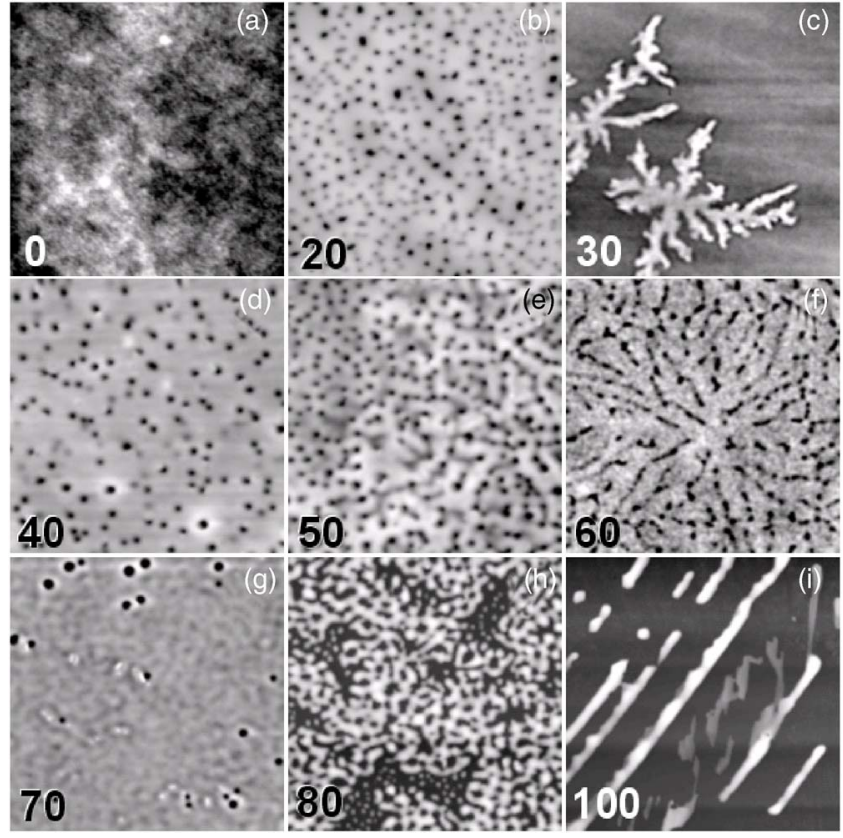

FIG. 4. The AFM images of STO/TPD blend films, at $\Phi=0 \%$ (a), $20 \%$ (b), $30 \%$ (c), $40 \%$ (d), 50\% (e), 60\% (f), 70\% (g), 80\% (h) and 100\% (i) (concentration values also reported at the bottom left corner of the images). All scan ranges are $15 \times 15 \mu \mathrm{m}^{2}$ but in (i), where a $30 \times 30 \mu \mathrm{m}^{2}$ area has been represented. The height scale ranges have been set so to maximize the contrast in each image, and are 1 (a), 60 (b), 25 (c), 60 (d), 70 (e), 4 (f), 25 (g), 95 (h) and $350 \mathrm{~nm}(\mathrm{i})$.

valleys now begin to form between adjacent holes, tending to link them. For $\Phi=60 \%$ [Fig. 4(f)] this reorganization forms extended grooves, aligned either along straight lines (see borders up, right and down in the image), or towards central points (see the starlike structure in the middle). Further, at $\Phi=70 \%$ [Fig. 4(g)] coalescence into larger voids occurs, which shows a uniform film around them. At even larger STO concentrations the blend seems to reach its limit of miscibility, and elevated ripples arise on the bare substrate for $\Phi=80 \%$ [Fig. 4(h)]. Finally, at $\Phi=90 \%$ (image not reported) and even more at $\Phi=100 \%$ [Fig. 4(i)] most blend material clusterizes into large and thick stripes (mean height around $300 \mathrm{~nm}$ ). These stripes look occasionally oriented to some direction, within local domains of several hundreds of micrometers whose origin is unclear. Some memory of the glass production process or of imperfect cleaning procedure, driving on a local scale the dewetting of the substrate in these extreme conditions, cannot be excluded.

\section{DISCUSSION}

In order to correlate the optical and the morphological properties of the blend films, the values of relevant parameters describing the surface topography have been extracted from AFM measurements, after averaging different sets of data from the same samples. In Fig. 5(a) the root-meansquare (rms) roughness and the dominant length scale ${ }^{21}$ versus $\Phi$ have been reported. Both the roughness (solid circles) and the dominant length (open circles) show a minimum very close to zero for $\Phi=0$, and reach the maximum values at the highest STO concentrations ( $\Phi=90$ and $100 \%)$, where the large and high stripes, likely due to phase segregation, appear [see Fig. 4(i)]. On the other hand, at the intermediate concentrations around which the crystal structures form, the roughness is not significantly different from the surrounding values, whereas the dominant length exhibits a peak. This is due to the flatness of the crystal islands [see Fig. 4(c)] as compared to the, likely amorphous, stripes of Fig. 4(i).

However, as the exciplex PL emission is expected to arise from the regions of homogeneous blend material, the most interesting morphological measure is the surface coverage of the blend film, which is reported in Fig. 5(b). In the estimation of this quantity, whenever three height levels were observed in the sample upon scratching it, we assumed the intermediate level to be the homogeneous blend film, and the topmost level to form a separated phase. In the case of crystallinelike islands [Fig. 4(c)] this designation appears obvious, while in the case of amorphouslike stripes [Fig. 4(i)] it was justified by selective dissolution ${ }^{22}$ of STO in methanol ${ }^{23}$ and by previous near-field optical measurements. ${ }^{24}$ As a result, we see that the profile of coverage in Fig. 5(b) shows a strong decrease at very high $\Phi$ values at which phase separation definitely occurs, and a local minimum at $\Phi=30 \%$ where the crystalline islands subtract surface area from the blend film.

In spite of this decrease of useful blend film area, the macroscopically averaged PL exciplex emission from the samples shows a maximum exactly at the same value of $\Phi$. The apparent contradiction can be understood in terms of efficiency of the blend film area. As we mentioned in Sec.
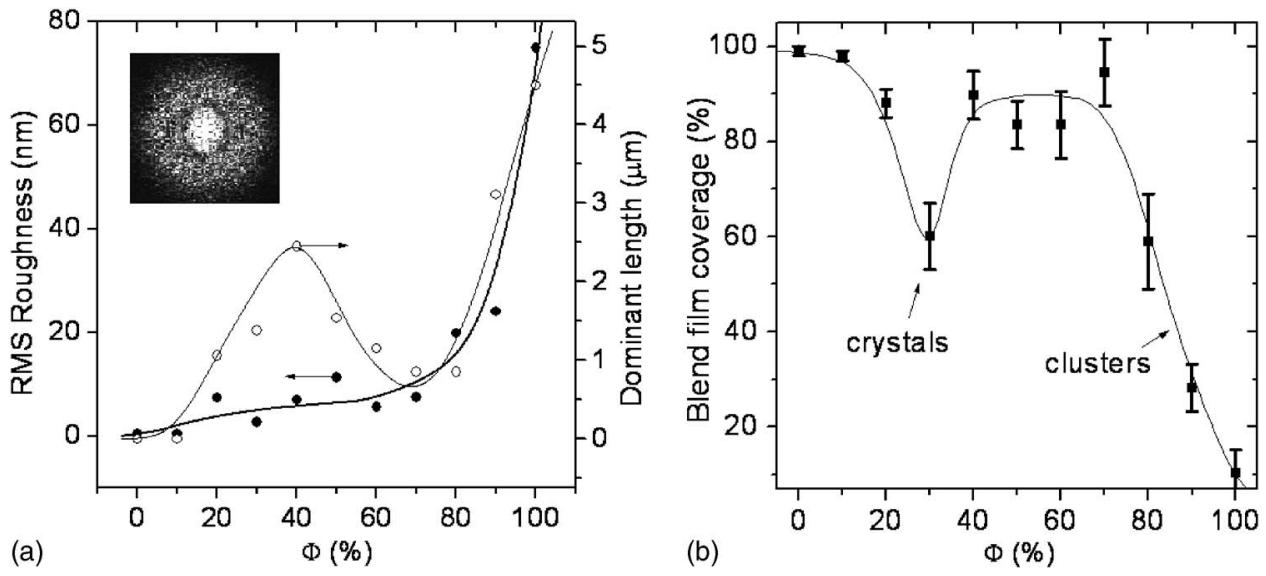

FIG. 5. (a) rms roughness and dominant length characteristics of the $\mathrm{STO} /$ TPD films in Fig. 4. (b) Blend film coverage of the same samples. In all cases the lines are just guides to the eye. Inset in (a) is the power spectrum of the AFM image in Fig. 4(h). 


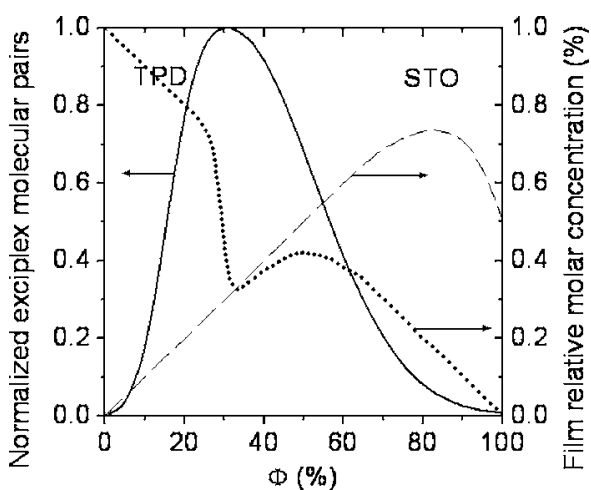

FIG. 6. Real model of the blend film system: the same as in Fig. 3(b), modified to account for the crystallization (TPD concentration profile pit) and phase separation (STO concentration profile dip at high $\Phi$ ) observed in the AFM images of Fig. 4.

III, the maximum efficiency of the film is expected when it contains the same number of molecules of the two species, such that the probability of a heterogeneous molecular pair forming an exciplex is maximized. Whereas in the ideal model [see Fig. 3(b)] this occurs for $\Phi=50 \%$, in the real case of subtraction of one blend component from the film one can have a relative molar concentration in the film that is different from the $\Phi$ of the originally spin-coated blend solution. Therefore, the 1:1 balance of the two molecular species in the emitting area can occur for a $\Phi$ value different from $50 \%$. In particular, a shift of the peak PL intensity towards $\Phi$ values lower than $50 \%$ is only justified if the blend component subtracted due to crystallization in Fig. 4(c) is TPD. In this case, as described in the modified model of Fig. 6, the negative peak in the TPD concentration profile (dotted line) allows the TPD concentration to equal that of STO for $\Phi$ lower than $50 \%$.

In order to support the hypothesis that the phaseseparated crystalline islands of Fig. 4(c) consisted of TPD and not STO, we checked this against selective dissolution. Whereas it was not possible to find a TPD selective solvent, our assumption was indirectly confirmed by the negative response of the structures to dissolution in methanol, which is selective for STO. Moreover, identification of the crystalline islands as TPD is also plausible due to the relatively low glass transition temperature $\left(63^{\circ} \mathrm{C}\right)$ of TPD.

Finally, the consistency of the model was checked by estimating the relative volumes of the structures in Fig. 4(c) against the TPD material supposedly subtracted from the blend film. Based on the consideration that neither TPD (molecular formula $\mathrm{C}_{38} \mathrm{H}_{32} \mathrm{~N}_{2}$ ) nor STO (molecular formula $\mathrm{C}_{10} \mathrm{H}_{20} \mathrm{Si}_{2} \mathrm{O}_{2}$ ) contains any high-density metal atom, we assumed the overall densities of the two blend components to be similar. Therefore, if it is further assumed that the degree of packing of the molecules into homogeneous solid-state films is also similar, the TPD and STO molar volumes should be roughly in the same ratio as the respective molecular weights, that is about a factor of 2 . With this in mind, we measured the relative volumes of the different regions of the sample in Fig. 4(c), namely, the elevated crystalline islands (surface coverage $40 \%$, thickness about $20 \mathrm{~nm}$, measured on the same image) and the surrounding blend film (surface coverage $60 \%$, thickness about $15 \mathrm{~nm}$, measured on a scratch elsewhere on the sample). ${ }^{23}$ From these considerations it turns out that the STO/TPD relative molar content in the blend film is about $40 \%,{ }^{25}$ which is closer, with respect to the relative concentration of the original solution of $\Phi=30 \%$, to the $50 \%$ value that gives the maximum number of exciplex molecular pairs in the film. The final deviation from $50 \%$ can arise from the fact that the low-energy crystalline structure of TPD is associated with a lower molar volume than in the amorphous form, i.e., a higher molecular content for the same volume, such that our model is underestimating the relative STO/TPD film content.

\section{CONCLUSIONS}

Improvement of OLEDs relies on tuning the properties of the active layers. In order to characterize the fabricated devices and provide information for efficient design, a clear correlation between morphological and emission properties is desirable. In this work we have focused on an organic blend of interest for lighting applications, and bridged the results of a characterization of photoluminescence to an AFM morphological analysis. In this frame, starting from the combined analysis of PL spectra and AFM height images, occurrence of complex phenomena such as crystallization and phase separation into amorphous clusters has been observed and interpreted successfully at a qualitative level. We plan to proceed further in the near future, by investigating the aging effects which are of technological interest for the stability of devices, also with the help of advanced morphological tools such as integral geometry.

\section{ACKNOWLEDGMENT}

The authors thank G. Barbarella (ICoCEA Bologna) for the STO.

${ }^{1}$ P. E. Burrows and S. R. Forrest, Appl. Phys. Lett. 64, 25 (1994).

${ }^{2}$ V. G. Kozlov, P. E. Burrows, G. Parthasarathy, and S. R. Forrest, Appl. Phys. Lett. 74, 1057 (1999).

${ }^{3}$ J. Shinar, Organic Light-Emitting Devices: A Survey (Springer, Berlin, 2003).

${ }^{4}$ R. S. Deshpande, V. Bulović, and S. R. Forrest, Appl. Phys. Lett. 75, 888 (1999).

${ }^{5}$ C. W. Ko and Y. T. Tao, Appl. Phys. Lett. 79, 4234 (2001).

${ }^{6}$ K. O. Cheon and J. Shinar, Appl. Phys. Lett. 84, 1201 (2004).

${ }^{7}$ The Exciplex, edited by M. Gordon and W. R. Ware (Academic, New York, 1975).

${ }^{8}$ C.-I. Chao and S.-A. Chen, Appl. Phys. Lett. 73, 426 (1998).

${ }^{9}$ C.-H. Kim and J. Shinar, Appl. Phys. Lett. 80, 2201 (2002).

${ }^{10}$ Y.-S. Huang, J.-H. Jou, W.-K. Weng, and J.-M. Liu, Appl. Phys. Lett. 80, 2782 (2002).

${ }^{11}$ M. Mazzeo et al., Appl. Phys. Lett. 82, 334 (2003).

${ }^{12}$ H. Ramanarayan and T. A. Abinandanan, Bull. Mater. Sci. 26, 189 (2003).

${ }^{13}$ H. Ade, D. A. Winesett, A. P. Smith, S. Qu, S. Ge, J. Sokolov, and M. Rafailovich, Europhys. Lett. 45, 526 (1999).

${ }^{14}$ S. Minko, M. Müller, D. Usov, A. Scholl, C. Froeck, and M. Stamm, Phys. Rev. Lett. 88, 035502-1 (2002).

${ }^{15}$ A. Bernasik, J. Włodarczyk-Miśkiewicz, W. Łużny, K. Kowalski, J. Raczkowska, J. Ryszc, and A. Budkowski, Synth. Met. 144, 253 (2004).

${ }^{16}$ M. Mazzeo, D. Pisignano, L. Favaretto, G. Barbarella, R. Cingolani, and G. Gigli, Synth. Met. 139, 671 (2003).

${ }^{17}$ G. Barbarella, O. Pudova, C. Arbizzani, M. Mastragostino, and A. Bongini, J. Org. Chem. 63, 1742 (1998).

${ }^{18}$ G. Barbarella et al., Synth. Met. 115, 47 (2000).

${ }^{19}$ J. Thompson, R. I. R. Blyth, M. Mazzeo, M. Anni, G. Gigli, and R. 
Cingolani, Appl. Phys. Lett. 79, 560 (2001).

${ }^{20}$ M. Mazzeo, D. Pisignano, L. Favaretto, G. Sotgiu, G. Barbarella, R. Cingolani, and G. Gigli, Synth. Met. 139, 675 (2003).

${ }^{21}$ In order to determine the dominant length, the two-dimensional power spectra of the AFM images have been first calculated [see, e.g., inset of Fig. 4(h)]. Then the radial profiles have been averaged into a single curve. The peak position $k_{\max }$ of this curve has been detected and the dominant length calculated as $1 / k_{\max }$. For nonisotropic sample morphologies as in Fig. 4(i), we used the value of $k_{\max }$ resulting from averaging over different regions of the sample, which exhibited domains with preferential direction randomly oriented on a larger scale.
${ }^{22}$ S. Walheim, M. Böltau, J. Mlynek, G. Krausch, and U. Steiner, Macromolecules 30, 4995 (1997).

${ }^{23}$ Supporting data available on request.

${ }^{24}$ M. Salerno, M. Mazzeo, M. C. Frassanito, S. Patanè, R. Cingolani, and G. Gigli, Appl. Phys. Lett. 86, 081907 (2005).

${ }^{25}$ If $\Phi_{f}$ is the relative molar concentration of the blend film, and we suppose that the crystal islands are only made of TPD, it turns out that $\Phi_{f}$ $=\Phi\left[C h_{f}+(1-C) h_{c}\right] /(1+\Phi) C h_{f}$, where $C$ is the film coverage (normalized to 1 ), and $h_{f}$ and $h_{c}$ are the thicknesses of the film layers and the crystal islands, respectively. 